\title{
Neural Correlates of Developmental Coordination Disorder: The Mirror Neuron System Hypothesis
}

\author{
Julie M. Werner", Sharon A. Cermak, Lisa Aziz-Zadeh \\ Division of Occupational Science \& Occupational Therapy, University of Southern California, Los Angeles, USA \\ Email: *jmwerner@usc.edu
}

Received January 13, 2012; revised January 29, 2012; accepted February 22, 2012

\begin{abstract}
Primary impairments of developmental coordination disorder (DCD) include impairments in motor skill, motor learning, and imitation. Such difficulties present challenges for individuals with DCD and may persist into adulthood, negatively impacting daily life in school, work, and social domains. A better understanding of the neural correlates of motor and imitation impairments in DCD holds the potential for informing development of treatment approaches to address these impairments. Although the disorder is assumed to be of neurological origin, little is known of the brain-based etiology of DCD. In recent years the discovery of a fronto-parietal circuit—known as the mirror neuron system—has enabled researchers to better understand imitation, general motor functions, and aspects of social cognition. Given its involvement in imitation and other motor functions, we propose that dysfunction in the mirror neuron system may underlie the characteristic impairments of DCD. We review literature pertaining to the mirror neuron system and develop a theory of disordered mirror neuron functioning in DCD. Finally, we review the limited neuroimaging literature available on neural correlates of DCD and show that the findings from those investigations are congruent with a mirror neuron system theory of DCD. Future research in this population should be designed to investigate specifically mirror neuron regions in individuals with DCD during skilled motor tasks and imitation in particular.
\end{abstract}

Keywords: Developmental Coordination Disorder; Dyspraxia; Imitation; Mirror Neuron System; Motor Learning

\section{Introduction}

Developmental coordination disorder is a neurologically based disorder broadly characterized by impaired motor coordination and impaired performance of daily activities requiring motor skill [1]. A similar and perhaps overlapping syndrome, developmental dyspraxia, has been described as impairment of motor planning and execution $[2,3]$ and is frequently tested with tasks requiring imitation [4,5]. Individuals with DCD or developmental dyspraxia may have difficulty learning and imitating skilled or sequenced movements, including object manipulation and tool use, assuming body postures, gesturing, and carrying out multi-step or goal-directed actions [4,6-11]. Functionally, individuals with DCD or developmental dyspraxia have trouble coordinating their movements to learn new fine and gross motor tasks required to perform instrumental daily activities, such as tying shoelaces, dressing, handwriting, playing sports, and using playground equipment $[1,3,12,13]$. In addition, decreased educational performance and poor social emotional adjustment, perhaps a result of participation limitations, have been found in children, adolescents, and adults with de-

${ }^{*}$ Corresponding author. velopmental coordination disorders [14-17].

Historically, a number of labels have been used in describing specific developmental disorders of motor function, such as clumsiness [18,19], physical awkwardness [20], minimal cerebral dysfunction [21], and congenital maladroitness (cited by [1] and [9]), with related descriptions like motor learning impairment [22], motor delay [22], motor control impairment [22], sensory integrative dysfunction [22] and hand-eye coordination impairment [22] sometimes found in the literature. In addition, dyspraxia is sometimes incorrectly used interchangeably with apraxia, a condition in which normal function is disrupted in adults or in previously typically developing children due to an upper motor neuron lesion (e.g., after a stroke; [9,21,23]). Although terminology varies, developmental dyspraxia is a term commonly used by occupational therapists and neurologists in the United States (observation by the authors), whereas DCD is a term widely used by various allied health and medical professionals, movement scientists, and related researchers internationally [24]. DCD is recognized as a diagnosable disorder by the American Psychiatric Association (APA) [25] when specific criteria are met: 1) motor coordination substantially below what is expected for chrono- 
logical age and intelligence; 2) motor difficulties interfere with activities of daily living or academic performance; 3) motor difficulties are not due to a general medical condition, such as cerebral palsy; and 4) motor difficulties are in excess of any mental retardation, if present. Although we will focus on DCD as suggested by an international terminology consensus [24], it is worthwhile also to include in our review the dyspraxia literature with the assumption that the two are likely overlapping with similar features and functional outcomes.

Although DCD is most apparent in childhood when many new skills are acquired and motor milestones are tracked, an increasing body of evidence reveals that many with the disorder continue to have difficulties with coordination and have reduced participation in motor tasks during adolescence and adulthood [15-17,26-29]. In a longitudinal study of children with DCD beginning when the children were 5 years old, Cantell, Smyth, and Ahonen [26] found that a subset of participants with relatively more severe motor impairments compared to other participants continued to have motor difficulties at age 17. Cousins and Smyth [16] documented poor performance on a variety of standardized motor tasks in 19 adults who qualified for a diagnosis of DCD as adults or were previously diagnosed as having DCD as children. Anecdotal descriptions by adolescents, young adults, and parents of young adults with probable DCD corroborate this data with reported sustained motor difficulties and lower performance in other life domains related to their motor impairments $[27,29]$. There is increasingly more research being conducted with adolescents and adults with DCD; however, only children have participated in the few available neuroimaging studies on the disorder. Conversely, the majority of neuroscience investigations using brain imaging methods in typically developing individuals have been performed with adult or young adult participants. There is somewhat limited data available for comparing the results of any neuroimaging study in children with DCD to typically developing children as well as limited evidence to explicate the neural correlates of DCD in adults and children.

Despite a number of recognizable impairments identified in this population [30,31], DCD is a disorder of largely unknown etiology. Furthermore, the neural correlates of DCD have not been investigated with advanced imaging methods to the extent that they have in adult apraxia [32-35] and other developmental disorders with motor impairments, such as cerebral palsy [36] and autism spectrum disorder [37]. Because impairments in imitation are common in individuals with DCD/dyspraxia $[4,10,38,39]$, we propose that a dysfunction in a fronto-parietal system may underlie the disorder. Known as the mirror neuron system (MNS), this fronto-parietal circuit is thought to be highly involved in imitation in humans $[40,41]$.
The putative human MNS, located in the inferior frontal gyrus (IFG), ventral premotor cortex (PMv), and inferior parietal lobule (IPL), is active during both action execution and action observation [42]. It is thought to be highly involved in action imitation as well as emulation [41]. In fact, evidence from a repetitive transcranial magnetic stimulation (rTMS) study indicated that disruption of the IFG in typically developing adults (aged 19 - 34) during an imitation task significantly interfered with imitation ability [43]. Therefore, hypothetically, individuals with developmental motor impairments may be limited in their ability to imitate, learn new movements, imagine movements, and produce goal-directed and object-oriented actions because they do not accurately perceive or match such actions with neural motor representations the way individuals without coordination disorders can. Mechanisms recruited in addition to MNS areas may be important for understanding the coding of visual input, imitation learning, and action goal understanding, all of which may contribute to imitative ability. We will review functions of the putative human MNS as it is involved in imitation, and propose that its dysfunction may be related to motor impairments in DCD.

\section{The Mirror Neuron System}

Researchers have argued that the MNS is a key element of human imitation [40,41,44-46]. Mirror neurons, originnally discovered in macaque monkeys using single-cell recording from surgically implanted microelectrodes, are a particular type of neuron that fire both when a monkey performs an action as well as when the monkey observes another individual performing a similar action [47-49]. In other words, the same neuron has both motor and sensory representations.

In monkeys, mirror neurons have been found in F5 and PF brain regions [48-50]. Based on gyri and sulci topography and evidence from cytoarchitecture, it is thought that the homologous region to F5 in humans is the pars opercularis of the inferior frontal gyrus [51,52]. Singleneuron recording studies are typically not conducted in humans for experimental purposes. Therefore, there is little direct evidence of the existence of mirror neurons at the cellular level [42,53] with the exception of Mukamel et al. [54], who reported mirror neurons in humans in the supplementary motor area, hippocampus, parahippo-campal gyrus, and entorhinal cortex when recording at sites in the medial frontal and temporal cortices. Brain imaging and neurophysiological studies have provided indirect evidence for a putative MNS in human frontal and parietal brain regions. Specifically, these areas include the IFG and adjacent PMv and the IPL (see Figure 1). (For a review of the brain imaging data and how these brain regions were determined to be functionally equivalent to monkey areas F5 and PF, see [42]). 


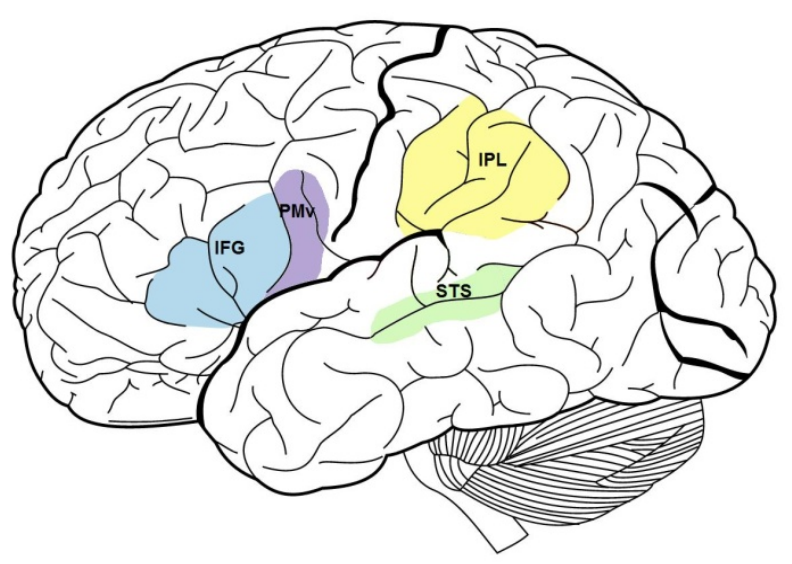

Figure 1. Lateral view of brain with frontal (IFG \& PMv) and parietal (IPL) mirror neuron system regions highlighted. The superior temporal sulcus is also labeled. [IFG = inferior frontal gyrus; $P M v$ = ventral premotor area; $I P L=$ inferior parietal lobule; STS = superior temporal sulcus].

\subsection{Properties of Mirror Neurons in Monkeys}

Properties of mirror neurons in the macaque brain are described here, as these data provide a framework for our understanding of the MNS. To elicit mirror neuron activity in the monkey, both observed and executed actions must be goal-directed (i.e., hand-object interaction or aiming for a target) [47-49,55]. This means that monkey mirror neurons are not merely responsive for a body part or an object alone, but code the conceptual goal of the action. Second, the majority of mirror neurons in F5 are broadly visuo-motor congruent; they respond to visually similar or conceptually related observed and executed actions. About a third of mirror neurons are strictly congruent in that the observed and executed actions have to match exactly, such as in the type of grasp used to hold an object [47-49]. Third, partial action sequences activate mirror neurons in monkeys who have previously seen the full action sequence, presumably because the animal infers the missing end sequence or goal of the action [56]. This property speaks to the point that mirror neurons are involved in action understanding through an internal motor representation of a full action, even when complete visual information is not immediately present [56]. Finally, many neurons in the monkey frontal mirror area resonate action information from audio as well as visual representation of object-related actions $[57,58]$. Together these findings in monkeys indicate that mirror neurons may aid in the integrated understanding of action information at a sensorimotor level rather than relying on conceptual interpretation through a semantic transformation process.

Although a number of distinct properties indicate that mirror neurons in monkeys function as a means of understanding the complex actions of others, there is con- flicting evidence on a monkey's ability to imitate [59-64]. Thus, imitation capability in humans supported by the MNS, as well as possible additional neural regions, probably represents an evolved mechanism from one that served more basic action understanding, such as that in monkeys. More evolved neural systems for learning may have facilitated imitation ability while the evolution of tool use may have helped select for imitation ability.

\subsection{The Human Mirror Neuron System and Imitation}

In recent years, a body of research primarily utilizing functional magnetic resonance imaging (fMRI) has provided evidence that the human MNS is active during action observation, execution, and imitation tasks [40,41,44, 46,65-67]. In a seminal study, Iacoboni et al. [40] monitored brain function in human frontal and parietal mirror regions while participants in an fMRI scanner were shown finger tapping actions or control stimuli. Participants passively observed the actions, imitated them, or executed a finger movement to a given cue. The researchers predicted that the inferior frontal gyrus and inferior parietal lobule, components of the putative human MNS, would follow an increasing pattern of signal activity. That is, these regions would be active during action observation, more for action execution, and when observation and execution are combined, as in the case of imitation, the highest signal intensity would be observed. The data validated these hypotheses [40].

Research using similar imitation tasks or tasks including object interactions has supported the previous findings that the fronto-parietal mirror network is most active during imitation when compared to observation and execution [66-70]. Furthermore, a re-analysis of data from Iacoboni et al.'s [40] study and six others from the same laboratory revealed additional details regarding functional segregation within the IFG for action observation and imitation [71]. This re-analysis indicated that the dorsal pars opercularis within the IFG was specifically active during action observation and imitation, with the most activation during imitation [71]. In addition, disruption of the IFG in either hemisphere with rTMS resulted in a transient impairment of imitation compared to a control movement task, indicating that this region is essential for imitation processing [43].

Together this evidence strongly indicates that these regions have a prominent role in typical human imitation, rendering the fronto-parietal mirror network a likely location of differences in neural activation between individuals with and without DCD. In particular, one may expect decreased signal intensity to correspond with imitation impairments. Although the vast majority of the MNS and imitation literature includes only adult participants, a few investigations have included typically de- 
veloping children (such as when comparing to children with ASD) and have reported MNS functioning similar to what has been described in typical adults [72-77]. Such evidence supports the view that a neural mirroring mechanism is present from childhood and that dysfunction in the MNS may contribute to developmental disorders of imitation, motor, and social skills [69].

\subsection{Coding of Biologic Actions in the Superior Temporal Sulcus}

Although not considered a mirror area because it is not active during action execution, the superior temporal sulcus (STS; see Figure 1) has been implicated in studies assessing the neural mechanisms of imitation [40,78-80]. The anterior portion of the STS is thought to code visual stimuli for meaningful and goal-directed biological actions [81,82]. In addition, the STS and the fronto-parietal mirror circuit are thought to be connected via the arcuate fasciculus and two tracts running parallel to it $[41,45,83]$. Thus, it has been proposed that information from the visual cortices undergoes further processing for the visual aspects of observed action by the STS and is then sent to the parietal cortex, which codes the affordances of the action and its kinesthetic qualities [42] via the posterior lateral tract parallel to the arcuate fasciculus terminating in the IPL [83]. Next, it is relayed to the IFG where action goal coding occurs [42] via another parallel tract to the arcuate fasciculus: the anterior lateral tract [83].

\subsection{Laterality of the Mirror Neuron System}

The visuo-motor mirror system is thought to be bilateral [84], and hemispheric differences have been found when comparing the experience of being imitated as opposed to imitating another person. In a study to investigate these differences using positron emission tomography (PET), Decety et al. [80] found the left inferior parietal cortex, thought to be involved with integrating visual and motor information for sequential processing of goal-directed actions $[85,86]$, correlated with imitating others. The right inferior parietal cortex, thought to be involved in body awareness $[87,88]$, correlated with being imitated. Furthermore, in an experiment in which researchers applied rTMS to the inferior parietal lobule, participants were less accurate on a self-other discrimination task when a virtual lesion was created on the right as compared to the left [89]. These results point to a lateralization of the parietal mirror neuron area in particular and general differences between processing self- and otherrelated visual information. In addition, some have suggested that the fronto-parietal mirror network, especially in the right hemisphere, and cortical midline structures, such as the medial prefrontal cortex, anterior cingulate cortex, and precuneus, work together to process self- other distinctions [90-92] and that the experience of being imitated is an important aspect of learning to imitate [93].

If differences in inferior parietal regions exist between individuals with DCD and typically developing individuals during imitation or while being imitated, such differences could have implications for understanding the neural etiology of the disorder. Dysfunction in the left inferior parietal cortex may indicate that motor or imitation impairments are related to a specific deficit in visuomotor integration $[85,86]$, whereas dysfunction in the right inferior parietal cortex may indicate that impairments are due to body awareness and difficulty distinguishing self from others [89]. Hemispheric differences in the MNS, particularly in the posterior parietal cortex, bring to light only weakly supported theories about cerebral laterality in DCD. It has been suggested that left parietal lobe dysfunction may underlie DCD based on the known lesion location usually responsible for acquired apraxia $[94,95]$. Although it cannot be assumed that the mechanisms of acquired apraxia equate to those of developmental motor impairments (in which there is no notable brain lesion), Zwicker and colleagues [96] found differential activation in the left IPL in the direction of higher blood-oxegenation-level dependent (BOLD) signal in children with DCD compared with typically developing peers on a simple motor task. In a follow-up investigation [97], these same researchers found greater signal in the right IPL in typically developing children compared with peers with DCD during a motor learning task. More evidence is needed to elucidate the particularities of potential laterality differences to DCD, especially in the context of imitation and imitation learning.

\section{Imitation Learning}

An evident benefit of imitation is the potential for learning novel actions through the copying of actions from others, such as through demonstration of motor acts. Indeed, humans imitate more than any other animal, and much of how humans learn is through imitation [98]. To investigate the neural correlates of imitation learning, Buccino et al. [68] asked non-musician participants to observe guitar chords played by a guitarist and, after a pause, imitate each chord. The results revealed the IPL, posterior portion of the IFG, and adjacent premotor region were most active during imitation. During the pause, however, the middle frontal gyrus, thought to be involved in the spatial aspects of working memory, and other motor preparation areas became active in addition to the mirror neuron circuit. Based on these results, Buccino et al. concluded that the MNS plays a prominent role in learning by imitation, but other regions, such as the middle frontal gyrus, may be needed as well to orchestrate the selection and organization of motor repre- 
sentations needed to learn and imitate a new motor program. Thus, regions beyond the MNS may also be implicated in DCD/dyspraxia.

In a study by Cross, Hamilton, and Grafton [99], expert dancers learned a complex, whole-body dance sequence over a period of five weeks, and researchers recorded their brain activity while watching the rehearsed and control sequences at the end of each week. Participants were asked to imagine themselves performing the movement sequences they observed and rate their perceived ability to perform each. Greater activation was found in the MNS, namely the left IPL and PMv, when participants observed rehearsed movement sequences they judged they could perform well, compared to control movement sequences for which they deemed their performance ability would be poor [99]. Although the participants in this study could not physically imitate the dance sequences while inside the fMRI scanner, the use of imagined movement is commonly used in brain imaging experiments to induce internal representation of motor plans without overt movement.

In a related study, Cross et al. [100] demonstrated that neural representations of movement sequences could be acquired by non-dancer participants either physically rehearsing or, to a lesser degree, passively observing actions. The experiment yielded activity in the premotor and inferior parietal regions for physical and observational training, with the dorsolateral prefrontal cortex additionally active for both types of rehearsal and the cerebellum involved during observation. These results, together with those of Buccino et al. [68] and Cross et al. [99], convey a consistent pattern of MNS activity when participants learned and imitated, or mentally simulated, new movements. However, additional brain regions related to a broad range of sensorimotor tasks appear to be necessary supplements to the core imitation circuit when learning new actions. Because DCD/dyspraxia entails impairment in learning new motor programs, these additional structures (i.e., prefrontal cortex, middle frontal cortex, anterior medial cortex, supplementary motor area, and superior parietal lobule) are important points of consideration for future investigations of the neurologic basis of DCD.

\section{Imitation, Empathy, \& the Mirror Neuron System}

The shared neural representation for action and perception which is thought to underlie imitation and action understanding, the MNS, is also thought to be involved in empathy [41,101-103]. Convincing evidence for this hypothesis comes from experiments showing that self-reported trait empathy and perspective-taking ability (usually measured with a widely-used empathy scale, the Interpersonal Reactivity Index; [104]) correlates with activity in the MNS [105-111]. In addition, in conditions in which empathy is known to be impaired, such as autism spectrum disorder (ASD), decreased activity in MNS regions has been found to correlate with social symptom severity [112]. Interestingly, individuals with ASD are also thought to have impairments in imitation [113-118].

Evidence points to higher rates of poorer psychosocial adjustment and decreased social skills and empathy in children and adults with DCD/dyspraxia compared to their peers without motor coordination problems [26,119-125]. There is no evidence in the literature to indicate the direction of this relationship. However, given the hypothesis that MNS dysfunction underlies imitation impairments, another question is how imitation ability, empathy, and activity in the MNS regions during imitation interact in individuals with DCD compared with their typically developed peers.

\section{Existing Evidence of Brain-Based Differences in DCD}

To date, no published fMRI studies have been conducted which examined imitation and MNS functioning in children or adults with dyspraxia, DCD, clumsiness, or other similarly labeled developmental motor coordination disorder. However, a limited number of brain imaging studies have broadly investigated group-level differences in brain activation on motor tasks between individuals with DCD and typically developing (TD) individuals [96,97, $126,127]$ and are fairly encouraging that a mirror system hypothesis may be supported.

Kashiwagi et al. [126] examined direct perceptual-motor mechanisms of DCD via a study of brain activation during a visuomotor tracking task in which participants followed an on-screen moving target with a joystick. The authors report less posterior parietal activation in the DCD group; however, these results appear to be undermined by a skewed results distribution as a consequence of one extreme outlier in the DCD group.

Querne et al. [127] examined attention, a corollary of developmental coordination disorder, by examining the attentional brain network in children with DCD and a control group using a go/no-go task in which participants responded when consecutive letters were presented (go) with the exception of " $\mathrm{X}$ " (no go). Using structural equation modeling to determine effective connectivity, the authors concluded that middle frontal and anterior cingulate cortex to inferior parietal cortex connectivity was increased in children with DCD, indicating less effective switching between go and no-go tasks in children with DCD and the need for additional recruitment of inhibittory brain responses to compensate.

Of more potential use in understanding the neurologycal etiology of DCD are a series of brain imaging experiments conducted by Zwicker and colleagues [96,97]. 
Using fMRI to measure whole-brain activation patterns in 7 children with DCD (aged 8 - 12 years) and 7 agematched TD peers, Zwicker et al. [96] predicted groupwise differences in cerebellar activity when participants performed a fine motor task adapted from the Movement Assessment Battery for Children [128] consisting of trail tracing using a joystick. Although these researchers did not find any significant difference between groups on task performance in the scanner, whole brain exploratory analysis revealed diffuse between-groups differences in a number of brain regions. Significant differences were observed in the left IPL and right supramarginal gyrus (DCD > TD) and left IFG and left precuneus (TD > DCD). In addition, a small cluster of activity was found in the right cerebellar lobule VI that was significantly greater in the DCD than TD group. The authors interpret these results in light of a theory that children with DCD may rely on visuospatial feedback in lieu of somatosensory feedback to guide their movements. Because the experimental task did not include imitation, action observation, or even the presence of another person, no conclusions can be drawn that these differences relate to a mirroring mechanism. However, the results are noteworthy because they include key MNS regions and may be indicative of different functioning in these regions.

In a follow-up study with the same participants as their previous investigation, Zwicker et al. [97] broadly hypothesized that the cerebellum, prefrontal cortex, and posterior parietal cortex would differ between DCD and TD groups on a retention test following practice of a fine motor task. Presumably their predictions were derived from a broad array of hypotheses implying large-scale motor and motor-related regional differences in DCD [95]. The same trail tracing task used in their previous study was employed and brain activity was measured at baseline and after three days of practice outside the scanner. In this study, group-wise differences from the initial learning task to retention were found in the bilateral IPL and other regions (DCD $<$ TD). As in the previous study conducted by Zwicker and colleagues, the procedures of this investigation do not provide grounds for making any assumptions about a mirroring mechanism, but still support the potential for broad functional differences in the primary MNS regions when comparing individuals with DCD and TD.

\section{Conclusion}

From the limited neuroimaging experiments in DCD, it is apparent there is a need to conduct more functional imaging studies to further examine direct neural correlates of DCD. In particular, this should be done in regard to MNS areas and their relationship to imitation and motor learning. Furthermore, condition-dependent differential activation (e.g., with novel gestures and during different time points of action) in regions of the human MNS should be compared between typically developing controls and participants with DCD to inform the contribution of each region to motor planning, motor learning, and imitation in DCD. Given the evidence that the human MNS is highly involved in empathy and social cognition and that individuals with DCD may have impairments in this area, it is also important to determine how traits like empathy may correlate with any differences in function of the mirror system regions in individuals with DCD. Examinations of these types using functional neuroimaging are crucial for increasing the current understanding of neuropathology in DCD.

\section{Acknowledgements}

J.M.W. would like to thank Kathryn Parks and Amanda Foran for their help in editing earlier versions of this manuscript.

\section{REFERENCES}

[1] S. Cermak, S. S. Gubbay and D. Larkin, "What Is Developmental Coordination Disorder?” In: Cermak \& Larkin, Eds., Developmental Coordination Disorder, Thomson Learning, Inc., 2002, pp. 2-22.

[2] J. O’Brien, J. Spencer, J. Atkinson, O. Braddick and J. Wattam-Bell, "Form and Motion Coherence Processing in Dyspraxia: Evidence of a Global Spatial Processing Deficit,” Neuroreport, Vol. 13, No. 11, 2002, pp. 1399-1402. doi:10.1097/00001756-200208070-00010

[3] Reeves and Cermak. "Disorders of Praxis," In: A. C. Bundy, S. J. Lane and E. A. Murray, Eds., Sensory Integration: Theory and Practice, 2nd Edition, F. A. Davis Company, 2002, pp. 71-100.

[4] A. J. Ayres, "Sensory Integration and Praxis Tests: Manual,” Western Psychological Services, Los Angeles, 19892004.

[5] P. H. Wilson, "Practitioner Review-Approaches to Assessment and Treatment of Children with DCD: An Evaluative Review," Journal of Child Psychology and Psychiatry, Vol. 46, No. 8, 2005, pp. 806-823. doi:10.1111/j.1469-7610.2005.01409.x

[6] A. J. Ayres, "Patterns of Perceptual Motor Dysfunction in Children: A Factor-Analytic Study,” Perceptual and Motor Skills, Vol. 20, No. 2, 1965, pp. 335-368. doi:10.2466/pms.1965.20.2.335

[7] M. Biancotto, A. Skabar, M. Bulgheroni, M. Carrozzi, and S. Zoia, "Neuromotor Deficits in Developmental Coordination Disorder: Evidence from a Reach-to-Grasp Task," Research in Developmental Disabilities, Vol. 32, No. 4, 2011, pp. 1293-1300. doi:10.1016/j.ridd.2011.02.007

[8] D. Dewey, “What Is Developmental Dyspraxia?” Brain and Cognition, Vol. 29, No. 3, 1995, pp. 254-273. doi:10.1006/brcg.1995.1281

[9] J. L. Poole, J. Gallagher, J. Janosky and C. Qualls, “The 
Mechanisms for Adult-Onset Apraxia and Developmental Dyspraxia: An Examination and Comparison of Error Patterns," American Journal of Occupational Therapy, Vol. 51, No. 5, 1997, pp. 339-346. doi:10.5014/ajot.51.5.339

[10] C. Sinani, D. A. Sugden and E. L. Hil, "Gesture Production in School Vs Clinical Samples of Children with Developmental Coordination Disorder (DCD) and Typically Developing Children," Research in Developmental Disabilities, Vol. 32, No. 4, 2011, pp. 1270-1282. doi:10.1016/j.ridd.2011.01.030

[11] K. Wilmut, J. P. Wann and J. H. Brown, "Problems in the Coupling of Eye and Hand in the Sequential Movements of Children with Developmental Coordination Disorder," Child: Care, Health and Development, Vol. 32, No. 6, 2006, pp. 665-678. doi:10.1111/j.1365-2214.2006.00678.x

[12] F. Gheysen, H. Van Waelvelde and W. Fias, "Impaired Visuo-Motor Sequence Learning in Developmental Coordination Disorder," Research in Developmental Disabilities, Vol. 32, No. 2, 2011, pp. 749-756. doi:10.1016/j.ridd.2010.11.005

[13] C. Missiuna and H. Polatajko, "Developmental Dyspraxia by Any Other Name: Are They All Just Clumsy Children?” American Journal of Occupational Therapy, Vol. 49, No. 7, 1995, pp. 619-627.

[14] Y. W. Chen, M. H. Tseng, F. C. Hu and S. A. Cermak, "Psychosocial Adjustment and Attention in Children with Developmental Coordination Disorder Using Different Motor Tasks," Research in Developmental Disabilities, Vol. 30, No. 6, 2009, pp. 1367-1377. doi:10.1016/j.ridd.2009.06.004

[15] M. Cantell and L. Kooistra, "Long-Term Outcomes of Developmental Coordination Disorder,” In: S. A. Cermak and D. Larkin, Eds., Developmental Coordination Disorder, Thomson Learning, Inc., 2002, pp. 23-38.

[16] M. Cousins and M. M. Smyth, "Developmental Coordination Impairments in Adulthood," Human Movement Science, Vol. 22, No. 4-5, 2003, pp. 433-459. doi:10.1016/j.humov.2003.09.003

[17] A. Kirby, D. Sugden, S. Beveridge and L. Edwards, "Developmental Co-Ordination Disorder (DCD) in Adolescents and Adults in Further and Higher Education,” Journal of Research in Special Education Needs, Vol. 8, No. 3, 2008, pp. $120-131$. doi:10.1111/j.1471-3802.2008.00111.x

[18] S. S. Gubbay, E. Ellis, J. N. Walton and S. D. M. Court, "Clumsy Children: A Study of Apraxic and Agnostic Defects in 21 Children,” Brain, Vol. 88, No. 2, 1969, pp. 295-312. doi:10.1093/brain/88.2.295

[19] M. T. Dare and N. Gordon, "Clumsy Children: A Disorder of Perception and Motor Organization," Developmental Medicine and Child Neurology, Vol. 12, No. 2, 1970, pp. 178-185. doi:10.1111/j.1469-8749.1970.tb01886.x

[20] A. E. Wall, G. Reid and J. Paton, "The Syndrome of Physical Awkwardness,” In: G. Reid, Ed., Problems in Movement Control, Elsevier Science Publishers, Amsterdam, 1990, pp. 283-315.
[21] S. A. Goodgold-Edwards and S. A. Cermak, "Integrating Motor Control and Motor Learning Concepts with Neuropsychological Perspectives on Apraxia and Developmental Dyspraxia," American Journal of Occupational Therapy, Vol. 44, No. 5, 1990, pp. 431-439.

[22] L. C. Magalhães, C. Missiuna and S. Wong, “Terminology Used in Research Reports of Developmental Coordination Disorder," Developmental Medicine and Child Neurology, Vol. 48, No. 11, 2006, pp. 937-941. doi:10.1017/S0012162206002040

[23] T. D. Sanger, D. Chen and M. R. Delgado, D. Gaebler-Spira, M. Hallett and J. W. Mink, "Definition and Classification of Negative Motor Signs in Childhood," Pediatrics, Vol. 118, 2006, pp. 2159-2167. doi:10.1542/peds.2005-3016

[24] H. J. Polatajko, M. Fox and C. Missiuna, “An International Consensus on Children with Developmental Coordination Disorder," Canadian Journal of Occupational Therapy, Vol. 62, No. 1, 1995, pp. 3-6.

[25] American Psychiatric Association, "Diagnostic and Statistical Manual of Mental Disorders: DSM-IV-TR,” American Psychiatric Association, Arlington, 2000.

[26] M. H. Cantell, M. M. Smyth and T. P. Ahonen, “Two Distinct Pathways for Developmental Coordination Disorder: Persistence and Resolution," Human Movement Science, Vol. 22, No. 4-5, 2003, pp. 413-431. doi:10.1016/j.humov.2003.09.002

[27] A. Kirby, L. Edwards and D. Sugden, "Emerging Adulthood in Developmental Co-Ordination Disorder: Parent and Young Adult Perspectives," Research in Developmental Disabilities, Vol. 32, No. 4, 2011, pp. 1351-1360. doi:10.1016/j.ridd.2011.01.041

[28] A. Kirby, L. Edwards, D. Sugden and S. Rosenblum, "The Development and Standardization of the Adult Developmental Co-Ordination Disorders/Dyspraxia Checklist (ADC)," Research in Developmental Disabilities, Vol. 31, No. 1, 2011, pp. 131-139. doi:10.1016/j.ridd.2009.08.010

[29] C. Missiuna, S. Moll, G. King, D. Stewart and K. MacDonald, "Life Experiences of Young Adults Who Have Coordination Difficulties," Canadian Journal of Occupational Therapy, Vol. 75, No. 3, 2008, pp. 157-166.

[30] H. Van Waelvelde, W. De Weerdt and P. De Cock, "Children with Developmental Coordination Disorder," European Bulletin of Adapted Physical Activity, Vol. 4, 2005, Electronic Journal.

[31] P. H. Wilson and B. E. McKenzie, "Information Processing Deficits Associated with Developmental Coordination Disorder: A Meta-Analysis of Research Findings," Journal of Child Psychology and Psychiatry, Vol. 39, No. 6, 1998, pp. 829-840. doi:10.1017/S0021963098002765

[32] L. J. Buxbaum, "Ideomotor Apraxia: A Call to Action," Neurocase, Vol. 7, No. 6, 2001, pp. 445-458. doi:10.1093/neucas/7.6.445

[33] G. Goldenberg, “Apraxia and the Parietal Lobes,” Neuropsychologia, Vol. 47, No. 6, 2009, pp. 1449-1459. doi:10.1016/j.neuropsychologia.2008.07.014

[34] K. Y. Haaland, D. L. Harrington and R. T. Knight, “Neu- 
ral Representations of Skilled Movement,” Brain, Vol. 123, No. 11, 2000, pp. 2306-2313. doi:10.1093/brain/123.11.2306

[35] M. Pazzaglia, N. Smania, E. Corato and S. M. Aglioti, "Neural Underpinnings of Gesture Discrimination in Patients with Limb Apraxia," The Journal of Neuroscience, Vol. 28, No. 12, 2008, pp. 3030-3041. doi:10.1523/JNEUROSCI.5748-07.2008

[36] M. Bax, C. Tydeman and O. Flodmark, "Clinical and MRI Correlates of Cerebral Palsy,” JAMA: The Journal of the American Medical Association, Vol. 296, No. 13, 2006, pp. 1602-1608. doi:10.1001/jama.296.13.1602

[37] S. H. Mostofsky, S. K. Powell, D. J. Simmonds, M. C. Goldberg, B. Caffo and J. J. Pekar, "Decreased Connectivity and Cerebellar Activity in Autism during Motor Task Performance,” Brain, Vol. 132, No. 9, 2009, pp. 2413-2425. doi:10.1093/brain/awp088

[38] D. Dewey, M. Cantell and S. G. Crawford, "Motor and Gestural Performance in Children with Autism Spectrum Disorders, Developmental Coordination Disorder, and/or Attention Deficit Hyperactivity Disorder," Journal of the International Neuropsychological Society, Vol. 13, No. 2, 2007, pp. 246-256. doi:10.1017/S1355617707070270

[39] S. Zoia, G. Pelamatti, M. Cuttini, V. Casotto and A. Scabar, "Performance of a Gesture in Children with and without DCD: Effect of Sensory Modalities," Developmental Medicine and Child Neurology, Vol. 44, No. 10, 2002, pp. 699-705. doi:10.1111/j.1469-8749.2002.tb00273.X

[40] M. Iacoboni, R. P. Woods, M. Brass, H. Bekkering, J. C. Mazziotta and G. Rizzolatti, "Cortical Mechanisms of Imitation,” Science, Vol. 286, No. 5449, 1999, pp. 25262528. doi:10.1126/science.286.5449.2526

[41] M. Acoboni, “Neural Mechanisms of Imitation,” Current Opinion in Biology, Vol. 15, 2005, pp. 632-636.

[42] G. Rizzolatti and L. Craighero, "The Mirror-Neuron System,” Annual Reviews Neuroscience, Vol. 27, 2004, pp. 169-192. doi:10.1146/annurev.neuro.27.070203.144230

[43] M. Heiser, M. Iacoboni, F. Maeda, J. Marcus and J. C. Mazziotta, "The Essential Role of Broca's Area in Imitation,” European Journal of Neuroscience, Vol. 17, No. 5, 2003, pp. 1123-1128. doi:10.1046/j.1460-9568.2003.02530.x

[44] M. A. Arbib, “The Mirror Neuron System, Imitation, and the Evolution of Language,” In: K. Dautenhahn and C. L. Nehaniv, Eds., Imitation in Animals and Artifacts, The MIT Press, Cambridge, 2002, pp. 229-280.

[45] G. Rizzolatti, L. Fogassi and V. Gallese, "Neurophysiological Mechanisms Underlying the Understanding and Imitation of Action," Nature Reviews Neuroscience, Vol. 2, 2001, pp. 661-669. doi:10.1038/35090060

[46] G. Rizzolatti, L. Fadiga, L. Fogassi and V. Gallese, "From Mirror Neurons to Imitation: Facts and Speculations,” In: A. N. Meltzoff and W. Prinz, Eds., The Imitative Mind, Cambridge University Press, Cambridge, 2002, pp. 247-266. doi:10.1017/CBO9780511489969.015

[47] G. Di Pellegrino, L. Fadiga, L. Fogassi and V. Gallese, "Understanding Motor Events: A Neuropsychological
Study,” Experimental Brain Research, Vol. 91, 1992, pp. 176-180.

[48] V. Gallese, L. Fadiga, L. Fogassi and G. Rizzolatti, “Action Recognition in the Pre-Motor Cortex," Brain, Vol. 119, No. 2, 1996, pp. 593-609. doi:10.1093/brain/119.2.593

[49] G. Rizzolatti, L. Fadiga, V. Gallese and L. Fogassi, "PreMotor Cortex and the Recognition of Motor Actions," Cognitive Brain Research, Vol. 3, No. 2, 1996, pp. 131141. doi:10.1016/0926-6410(95)00038-0

[50] V. Gallese, L. Fadiga, L. Fogassi and G. Rizzolatti, "Action Representation and the Inferior Parietal Lobule," Common Mechanisms in Perception and Action Attention and Performance, Vol. 19, 2002, pp. 247-266.

[51] M. Petrides and D. N. Pandya, "Comparative Architectonic Analysis of the Human and the Macaque Frontal Cortex,” In: F. Boller and J. Grafman, Eds., Handbook of Neuropsychology, Vol. IX, Elsevier, New York, 1997, pp. 17-58.

[52] G. Rizzolatti and M. Matelli, “Two Different Streams Form the Dorsal Visual System: Anatomy and Functions," Experimental Brain Research, Vol. 153, No. 2, 2003, pp. 146-157. doi:10.1007/s00221-003-1588-0

[53] G. Rizzolatti, "The Mirror Neuron System and Its Function in Humans," Anatomy and Embroyology, Vol. 210, No. 5-6, 2005, pp. 419-421. doi:10.1007/s00429-005-0039-z

[54] R. Mukamel, A. D. Ekstrom, J. Kaplan, M. Iacoboni and I. Fried, "Single-Neuron Responses in Humans during Execution and Observation of Actions," Current Biology, Vol. 20, No. 3, 2010, pp. 1-7. doi:10.1016/j.cub.2010.02.045

[55] D. Tkach, J. Reimer and N. G. Hatsopoulos, “Congruent Activity during Action and Action Observation in Motor Cortex,” Journal of Neuroscience, Vol. 27, No. 48, 2007, pp. 13241-13250. doi:10.1523/JNEUROSCI.2895-07.2007

[56] M. Umilta, E. Kohler, V. Gallese, L. Fogassi, L. Fadiga, C. Keysers and G. Rizzolatti, "I Know What You Are Doing: A Neurophysiological Study,” Neuron, Vol. 31, No. 1, 2001, pp. 155-165. doi:10.1016/S0896-6273(01)00337-3

[57] C. Keysers, E. Kohler, M. A. Umilta, L. Nanetti, L. Fogassi and V. Gallese, "Audiovisual Mirror Neurons and Action Recognition,” Experimental Brain Research, Vol. 153, No. 4, 2003, pp. 628-636. doi:10.1007/s00221-003-1603-5

[58] E. Kohler, C. Keysers, M. A. Umilta, L. Fogassi, V. Gallese and G. Rizzolatti, "Hearing Sounds, Understanding Actions: Action Representation in Mirror Neurons," Science, Vol. 297, No. 5582, 2002, pp. 846-848. doi:10.1126/science.1070311

[59] P. F. Ferrari, S. Rozzi and L. Fogassi, "Mirror Neurons Responding to Observation of Actions Made with Tools in Monkey Ventral Premotor Cortex,” Journal of Cognitive Neuroscience, Vol. 17, No. 2, 2005, pp. 212-226. doi:10.1162/0898929053124910

[60] F. Subiaul, J. F. Cantlon, R. L. Holloway and H. S. Terrace, "Cognitive Imitation in Rhesus Macaques,” Science, 
Vol. 305, No. 5682, 2004, pp. 407-410. doi:10.1126/science.1099136

[61] E. Visalberghi and D. Fragaszy, "Do Monkeys Ape?Ten Years after,” In: K. Dautenhahn and C. L. Nehaniv, Eds., Imitation in Animals and Artifacts, The MIT Press, Cambridge, 2002, pp. 471-500.

[62] B. Voelkl and L. Huber, "True Imitation in Marmosets," Animal Behavior, Vol. 60, No. 2, 2000, pp. 195-202. doi:10.1006/anbe.2000.1457

[63] A. Whiten, "Imitation of Sequential and Hierarchical Structure in Action: Experimental Studies with Children and Chimpanzees,” In: K. Dautenhahn and C. L. Nehaniv, Eds., Imitation in Animals and Artifacts, The MIT Press, Cambridge, 2002, pp. 191-210.

[64] A. Whiten and R. Ham, "On the Nature and Evolution of Imitation in the Animal Kingdom: Reappraisal of a Century of Research," Advances in the Study of Behavior, Vol. 21, 1992, pp. 239-283. doi:10.1016/S0065-3454(08)60146-1

[65] G. Buccino, F. Binkofski, G. R. Fink, L. Fadiga, L. Fogassi, V. Gallese and H.-J. Freund, "Action Observation Activates Premotor and Parietal Areas in a Somatotopic Manner: An fMRI Study," European Journal of Neuroscience, Vol. 13, 2001, pp. 400-404.

[66] P. L. Jackson, A. N. Meltzoff and J. Decety, "Neural Circuits Involved in Imitation and Perspective-Taking," Neuroimage, Vol. 31, No. 1, 2006, pp. 429-439. doi:10.1016/j.neuroimage.2005.11.026

[67] L. Koski, M. Iacoboni, M.-C. Dubeau, R. P. Woods and J. C. Mazziotta, "Modulation of Cortical Activity during Different Imitative Behaviors," Journal of Neurophysiology, Vol. 89, No. 1, 2003, pp. 460-471. doi:10.1152/jn.00248.2002

[68] G. Buccino, S. Vogt, A. Ritzl, G. R. Fink, K. Zilles, H.-J. Freund and G. Rizzolatti, "Neural Circuits Underlying Imitation Learning of Hand Actions: An Event-Related fMRI Study,” Neuron, Vol. 42, No. 2, 2004, pp. 323-334. doi:10.1016/S0896-6273(04)00181-3

[69] E. Kohler, C. Keysers, M. A. Umilta, L. Fogassi, V. Gallese and G. Rizzolatti, "Hearing Sounds, Understanding Actions: Action Representation in Mirror Neurons," Science, Vol. 297, No. 5582, 2002, pp. 846-848. doi:10.1126/science.1070311

[70] N. Nishitani and R. Hari, "Temporal Dynamics of Cortical Representation for Action," Proceedings of the $\mathrm{Na}$ tional Academies of Sciences of the United States of America, Vol. 97, No. 2, 2000, pp. 913-918. doi:10.1073/pnas.97.2.913

[71] I. Molnar-Szakacs, M. Iacoboni, L. Koski and J. C. Mazziotta, "Functional Segregation within Pars Opercularis of the Inferior Frontal Gyrus: Evidence from fMRI Studies of Imitation and Action Observation," Cerebral Cortex, Vol. 15, No. 7, 2005, pp. 986-994. doi:10.1093/cercor/bhh199

[72] M. Dapretto, M. S. Davies, J. H. Pfeifer, A. A. Scott, M. Sigman, S. Y. Bookheimer and M. Iacoboni, "Understanding Emotions in Others: Mirror Neuron Dysfunction in Children with Autism Spectrum Disorders," Nature Neuroscience, Vol. 9, No. 1, 2006, pp. 28-30.

\section{doi:10.1038/nn1611}

[73] J. Martineau, S. Cochin, R. Magne and C. Barthelemy, "Impaired Cortical Activation in Autistic Children: Is the Mirror Neuron System Involved?” International Journal of Psychophysiology, Vol. 68, No. 1, 2008, pp. 35-40. doi:10.1016/j.ijpsycho.2008.01.002

[74] N. Nishitani, S. Avikainen and R. Hari, “Abnormal Imitation-Related Cortical Activation Sequences in Asperger's Syndrome," Annals of Neurology, Vol. 55, No. 4, 2004, pp. 558-562. doi:10.1002/ana.20031

[75] L. M. Oberman, E. M. Hubbard, J. P. McCleery, E. L. Altschuler, V. S. Ramachandran and J. A. Pineda, "EEG Evidence for Mirror Neuron Dysfunction in Autism Spectrum Disorders," Cognitive Brain Research, Vol. 24, No. 2, 2005, pp. 190-198. doi:10.1016/j.cogbrainres.2005.01.014

[76] H. Théoret, E. Halligan, M. Kobayashi, F. Fregni, H. Tager-Flusberg and A. Pascual-Leone, "Impaired Motor Facilitation during Action Observation in Individuals with Autism Spectrum Disorders,” Current Biology, Vol. 15, No. 3, 2005, pp. R84-R85. doi:10.1016/j.cub.2005.01.022

[77] J. F. Lepage and H. Théoret, "The Mirror Neuron System: Grasping Others' Actions from Birth?” Developmental Science, Vol. 10, No. 5, 2007, pp. 513-523. doi:10.1111/j.1467-7687.2007.00631.x

[78] T. Chaminade, A. N. Meltzoff and J. Decety, "Does the End Justify the Means? A PET Exploration of the Mechanisms Involved in Human Imitation,” Neuroimage, Vol. 15, No. 2, 2002, pp. 318-328. doi:10.1006/nimg.2001.0981

[79] T. Chaminade, A. N. Meltzoff and J. Decety, “An fMRI Study of Imitation: Action Representation and Body Schema," Neuropsychologia, Vol. 43, No. 1, 2005, pp. 115-127. doi:10.1016/j.neuropsychologia.2004.04.026

[80] J. Decety, T. Chaminade, J. Grezes and A. N. Meltzoff, "A PET Exploration of the Neural Mechanisms Involved in Reciprocal Imitation,” Neuroimage, Vol. 15, No. 1, 2002, pp. 265-272. doi:10.1006/nimg.2001.0938

[81] J. Jellema, C. I. Baker, B. Wicker and D. I, Perrett, “Neural Representation for the Perception of the Intentionality of Actions," Brain and Cognition, Vol. 44, 2000, pp. 280-302. doi:10.1006/brcg.2000.1231

[82] D. I. Perrett, M. H. Harries, R. Bevan, S. Thomas, P. J. Benson, A. J. Mistlin and J. E. Ortega, "Frameworks of Analysis for the Neural Representation of Animate Objects and Actions,” Journal of Experimental Biology, Vol. 146, 1989, pp. 87-113.

[83] M. Catani, D. K. Jones, and D. H. Ffytche, "Perisylvian Language Networks of the Human Brain," Annals of Neurology, Vol. 57, No. 1, 2004, pp. 8-16. doi:10.1002/ana.20319

[84] L. Aziz-Zadeh, L. Koski, E. Zaidel, J. Mazziotta and M. Iacoboni, "Lateralization of the Human Mirror Neuron System,” The Journal of Neuroscience, Vol. 26, No. 11, 2006, pp. 2964-2970. doi:10.1523/JNEUROSCI.2921-05.2006

[85] P. K. Mutha, R. L. Sainberg and K. Y. Haaland, "Left Parietal Regions Are Critical for Adaptive Visuomotor 
Control,” Journal of Neuroscience, Vol. 31, No. 19, 2011, pp. 6972-6981. doi:10.1523/JNEUROSCI.6432-10.2011

[86] E. B. Torres, A. Raymer, L. J. G. Rothi, K. M. Heilman and H. Poizner, "Sensory-Spatial Transformations in the Left Posterior Parietal Cortex May Contribute to Reach Timing," Journal of Neurophysiology, Vol. 104, No. 5, 2010, pp. 2375-2388. doi:10.1152/jn.00089.2010

[87] G. Berlucchi and S. M. Aglioti, "The Body in the Brain: Neural Bases of Corporeal Awareness," Trends in Neuroscience, Vol. 20, No. 12, 1997, pp. 560-564. doi:10.1016/S0166-2236(97)01136-3

[88] A. Fotopoulou, A. Rudd, P. Holmes and M. Kopelman, "Self-Observation Reinstates Motor Awareness in Anosognosia for Hemiplegia,” Neuropsychologia, Vol. 47, No. 5, 2009, pp. 1256-1260. doi:10.1016/j.neuropsychologia.2009.01.018

[89] L. Q. Uddin, I. Molnar-Szakacs, E. Zaidel and M. Iacoboni, "rTMS to the Right Inferior Parietal Lobule Disrupts Self-Other Discrimination," Social Cognitive and Affective Neuroscience, Vol. 1, No. 1, 2006, pp. 65-71. doi:10.1093/scan/nsl003

[90] J. Decety and J. A. Sommerville, "Shared Representations between Self and Other: A Social Cognitive Neuroscience View,” Trends in Cognitive Sciences, Vol. 7, No. 12, 2003, pp. 527-533. doi:10.1016/j.tics.2003.10.004

[91] L. Q. Uddin, M. Iacoboni, C. Lange and J. Keenan, “The Self and Social Cognition: The Role of Cortical Midline Structures and Mirror Neurons," Trends in Cognitive Sciences, Vol. 11, No. 4, 2007, pp. 153-157. doi:10.1016/j.tics.2007.01.001

[92] L. Q. Uddin, J. T. Kaplan, I. Molnar Szakacs, E. Zaidel and M. Iacoboni, "Self-Face Recognition Activates a Frontoparietal 'Mirror' Network in the Right Hemisphere: An Event-Related fMRI Study,” Neuroimage, Vol. 25, No. 3, 2005, pp. 926-935. doi:10.1016/j.neuroimage.2004.12.018

[93] J. Nadel, "Imitation and Imitation Recognition: Functional Use in Preverbal Infants and Nonverbal Children with Autism,” In: A. N. Meltzoff and W. Prinz, Eds., The Imitative Mind, Cambridge University Press, Cambridge, 2002, pp. 42-62. doi:10.1017/CBO9780511489969.003

[94] M. K. Morris, “Developmental Dyspraxia,” In: L. J. G. Rothi and K. M. Heilman, Eds., Apraxia: The Neuropsychology of Action, Psychology Press, New York, 1997, pp. 245-268.

[95] J. G. Zwicker, C. Missiuna and L. A. Boyd, "Neural Correlates of Developmental Coordination Disorder: A Review of Hypotheses," Journal of Child Neurology, Vol. 24, No. 10, 2009, pp. 1273-1281. doi:10.1177/0883073809333537

[96] J. G. Zwicker, C. Missiuna, S. R. Harris and L. A. Boyd, "Brain Activation of Children with Developmental Coordination Disorder Is Different than Peers," Pediatrics, Vol. 126, 2010, pp. e678-e686. doi:10.1542/peds.2010-0059

[97] J. G. Zwicker, C. Missiuna, S. R. Harris and L. A. Boyd, "Brain Activation Associated with Motor Skill Practice on Children with Developmental Coordination Disorder: An fMRI Study,” International Journal of Developmental
Neuroscience, Vol. 29, No. 2, 2011, pp. 145-152. doi:10.1016/j.ijdevneu.2010.12.002

[98] W. Prinz and A. N. Meltzoff, "An Introduction to the Imitative Mind and Brain,” In: A. N. Meltzoff and W. Prinz, Eds., The Imitative Mind, Cambridge University Press, Cambridge, 2002, pp. 1-15. doi:10.1017/CBO9780511489969.001

[99] E. S. Cross, A. F. de C. Hamilton and S. T. Grafton, "Building a Motor Simulation De Novo: Observation of Dance by Dancers,” Neuroimage, Vol. 31, No. 3, 2006, pp. 1257-1267. doi:10.1016/j.neuroimage.2006.01.033

[100] E. S. Cross, D. J. M. Kraemer, A. F. de C. Hamilton, W. M. Kelley and S. T. Grafton, "Sensitivity of the Action Observation Network to Physical and Observational Learning,” Cerebral Cortex, Vol. 19, 2009, pp. 315-326. doi:10.1093/cercor/bhn083

[101] V. Gallese, “The 'Shared Manifold' Hypothesis: From Mirror Neurons to Empathy,” Journal of Consciousness Studies, Vol. 8, No. 5-7, 2001, pp. 33-50.

[102] V. Gallese, C. Keysers and G. Rizzolatti, “A Unifying View of the Basis of Social Cognition," Trends in Cognitive Science, Vol. 8, 2004, pp. 396-403. doi:10.1016/j.tics.2004.07.002

[103] M. Iacoboni, “Imitation, Empathy, and Mirror Neurons,” Annual Review of Psychology, Vol. 60, 2009, pp. 653-670. doi:10.1146/annurev.psych.60.110707.163604

[104] M. H. Davis, "Measuring Individual Differences in Empathy: Evidence for a Multidimensional Approach,” Journal of Personality and Social Psychology, Vol. 44, No. 1, 1983, pp. 113-126. doi:10.1037/0022-3514.44.1.113

[105] L. Aziz-Zadeh, T. Sheng and A. Gheytanchi, "Common Premotor Regions for the Perception and Production of Prosody and Correlations with Empathy and Prosodic Ability,” PLoS One, Vol. 5, 2010, p. e8759. doi:10.1371/journal.pone.0008759

[106] L. Carr, M. Iacoboni, M.-C. Dubeau, J. C. Mazziotta and G. L. Lenzi, "Neural Mechanisms of Empathy in Humans: A Relay from Neural Systems for Imitation to Limbic Areas," Proceedings of the National Academy of Sciences of the United States of America, Vol. 100, No. 9, 2003, pp. 5497-5502. doi:10.1073/pnas.0935845100

[107] V. Gazzola, L. Aziz-Zadeh and C. Keysers, “Empathy and the Somatotopic Auditory Mirror Neuron System in Humans," Current Biology, Vol. 16, No. 18, 2006, pp. 1824-1829. doi:10.1016/j.cub.2006.07.072

[108] J. T. Kaplan and M. Iacoboni, "Getting a Grip on Other Minds: Mirror Neurons, Intention Understanding, and Cognitive Empathy," Social Neuroscience, Vol. 1, No. 3-4, 2006, pp. 175-183. doi:10.1080/17470910600985605

[109] C. Lamm, C. D. Batson and J. Decety, “The Neural Substrate of Human Empathy: Effects of Perspective-Taking and Cognitive Appraisal," Journal of Cognitive Neuroscience, Vol. 19, No. 1, 2007, pp. 42-58. doi:10.1162/jocn.2007.19.1.42

[110] J. H. Pfeifer, M. Iacoboni, J. C. Mazziotta and M. Dapretto, “Mirroring Others' Emotions Relates to Empathy and Interpersonal Competence in Children," NeuroImage, Vol. 39, No. 4, 2008, pp. 2076-2085. 
doi:10.1016/j.neuroimage.2007.10.032

[111] M. Schulte-Ruther, H. J. Markowitsch, G. R. Fink and M. Piefke, "Mirror Neuron and Theory of Mind Mechanisms Involved in Face-to-Face Interactions: A Functional Magnetic Resonance Imaging Approach to Empathy," Journal of Cognitive Neuroscience, Vol. 19, No. 8, 2007, pp. 1354-1372. doi:10.1162/jocn.2007.19.8.1354

[112] M. Dapretto, M. S. Davies, J. H. Pfeifer, A. A. Scott, M. Sigman, S. Y. Bookheimer and M. Iacoboni, "Understanding Emotions in Others: Mirror Neuron Dysfunction in Children with Autism Spectrum Disorders," Nature Neuroscience, Vol. 9, No. 1, 2006, pp. 28-30. doi:10.1038/nn1611

[113] S. H. Mostofsky, P. Dubey, V. K. Jerath, E. M. Jansiewicz, M. C. Goldberg and M. B. Denckla, "Developmental Dyspraxia Is Not Limited to Imitation in Children With Autism Spectrum Disorders," Journal of the International Neuropsychological Society, Vol. 12, No. 3, 2006, pp. 314-326. doi:10.1017/S1355617706060437

[114] S. J. Rogers, S. L. Hepburn, T. Stackhouse and E. Wehner, "Imitation Performance in Toddlers with Autism and Those with Other Developmental Disorders," Journal of Child Psychology and Psychiatry, Vol. 44, No. 5, 2003, pp. 763-781. doi:10.1111/1469-7610.00162

[115] I. M. Smith and S. E. Bryson, "Imitation and Action in Autism: A Critical Review,” Psychological Bulletin, Vol. 116, No. 2, 1994, pp. 259-273. doi:10.1037/0033-2909.116.2.259

[116] J. M. Werner, L. Aziz-Zadeh and S. A. Cermak, "Praxis and Imitation in Children with Autism Spectrum Disorder,” NDTA Network, Vol. 18, 2011, pp. 10-15.

[117] J. H. G. Williams, A. Whiten and T. Singh, “A Systematic Review of Action Imitation in Autistic Spectrum Disorder," Journal of Autism and Developmental Disorders, Vol. 34, No. 3, 2004, pp. 285-299. doi:10.1023/B:JADD.0000029551.56735.3a

[118] J. H. G. Williams, G. D. Waiter, A. Gilchrist, D. I. Perrett, A. D. Murray and A. Whiten, "Neural Mechanisms of imitation and 'Mirror Neuron' Functioning in Autistic Spectrum Disorder,” Neuropsychologia, Vol. 44, No. 4, 2006, pp. 610-621. doi:10.1016/j.neuropsychologia.2005.06.010

[119] H.-F. Chen and E. S. Cohn, "Social Participation for Children with Developmental Coordination Disorder: Conceptual, Evaluation and Intervention Considerations,”
Physical and Occupational Therapy in Pediatrics, Vol. 23, No. 4, 2003, pp. 61-78. doi:10.1080/J006v23n04_05

[120] Y. W. Chen, M. H. Tseng, F. C. Hu and S. A. Cermak, "Psychosocial Adjustment and Attention in Children with Developmental Coordination Disorder Using Different Motor Tasks,” Research in Developmental Disabilities, Vol. 30, No. 6, 2009, pp. 1367-1377. doi:10.1016/j.ridd.2009.06.004

[121] A. Cummins, J. P. Piek and M. J. Dyck, "Motor Coordination, Empathy, and Social Behavior in School-Aged Children,” Developmental Medicine and Child Neurology, Vol. 47, 2005, pp. 437-442. doi:10.1017/S001216220500085X

[122] D. Dewey, B. J. Kaplan, S. G. Crawford and B. N. Wilson, "Developmental Coordination Disorder: Associated Problems in Attention, Learning, and Psychosocial Adjustment," Human Movement Science, Vol. 21, No. 5-6, 2002, pp. 905-918. doi:10.1016/S0167-9457(02)00163-X

[123] B. Kaplan, S. Crawford, M. Cantell, L. Kooistra and D. Dewey, "Comorbidity, Co-Occurrence, Continuum: What's in a Name?” Child: Care, Health and Development, Vol. 32, No. 6, 2006, pp. 723-731. doi:10.1111/j.1365-2214.2006.00689.x

[124] R. A. Skinner and J. P. Piek, "Psychosocial Implications of Poor Motor Coordination in Children and Adolescents,” Human Movement Science, Vol. 20, No. 1-2, 2001, pp.73-94. doi:10.1016/S0167-9457(01)00029-X

[125] J. Visser, "Developmental Coordination Disorder: A Review of Research on Subtypes and Comorbidities," $\mathrm{Hu}$ man Movement Science, Vol. 22, No. 4-5, 2003, pp. 479493. doi:10.1016/j.humov.2003.09.005

[126] M. Kashiwagi, S. Iwaki, Y. Narumi, H. Tamai and S. Suzuki, "Parietal Dysfunction in Developmental Coordination Disorder: A Functional MRI Study,” Brain Imaging, Vol. 20, No. 15, 2009, pp. 1319-1324.

[127] L. Querne, P. Berquin, M.-P. Vernier-Hauvette, S. Fall, L. Deltour, M.-E. Meyer and G. de Marco, "Dysfunction of the Attentional Brain Network in Children with Developmental Coordination Disorder: A fMRI Study,” Brain Research, Vol. 1244, 2008, pp. 89-102. doi:10.1016/j.brainres.2008.07.066

[128] S. E. Henderson and D. A. Sugden, "The Movement Assessment Battery for Children,” Harcourt, London, 1992. 